\section{On the Design of AQM Supporting TCP Flows Using Robust Control Theory}

\author{
Pierre-François Quet and Hitay Özbay
}

\begin{abstract}
Recently it has been shown that the active queue management schemes implemented in the routers of communication networks supporting transmission control protocol (TCP) flows can be modeled as a feedback control system. Based on a delay differential equations model of TCPs congestion-avoidance mode different control schemes have been proposed. Here a robust controller is designed based on the known techniques for $\mathcal{H}^{\infty}$ control of systems with time delays.
\end{abstract}

Index Terms-Active queue management (AQM), communication networks, control of uncertain systems with time delays, $\mathcal{H}^{\infty}$ control.

\section{INTRODUCTION}

Recently several mathematical models of active queue management (AQM) schemes supporting transmission control protocol (TCP) flows in communication networks have been proposed [1]-[3]. From these models a control theory-based approach can be used to analyze or to design AQM schemes. The authors of [2] have derived a delay differential equations model of TCPs congestion avoidance mode and further simplified this model focusing the design of a proportional-integral controller on the low-frequency dynamics, considering the high-frequency dynamics as parasitic. Their controller could guarantee some robustness with respect to the network parameters uncertainties. However, if the uncertainties to be tolerated for stability are "relatively" large, the system's response becomes sluggish. Motivated by their work, we design in this note an $\mathcal{H}^{\infty}$ controller for their original linear system, without neglecting high-frequency dynamics, that ensures robust stability and good performance for a wider range of network parameters uncertainties. As in [2], we assume that the AQM mechanism brings the system to the neighborhood of an equilibrium (operating point), so that we take the same linear model. Large deviations from this operating point (e.g., in the form of TCP time-out and slow-start phases, buffer overflow, empty queue) are ignored.

Other control theoretic based design of AQM include [4]-[6] while the importance of considering time delays is pointed out in [7]-[9] and a general overview of Internet congestion control literature can be found in [10].

\section{Mathematical Model of AN AQM Scheme SupPoRTING TCP FLOWS}

We consider in this note the network configuration consisting of a single router receiving $N$ TCP flows, we assume that the AQM scheme implemented at the router marks packets using explicit congestion notification (ECN) [11] to inform the TCP sources of impending congestion. In the following, we ignore the TCP slow start and time out

Manuscript received October 23, 2002; revised August 23, 2003. Recommended by Associate Editor C. D. Charalambous. This work was supported in part by the National Science Foundation under Grant ANI-0073725.

P.-F. Quet was with the Department of Electrical Engineering, The Ohio State University, Columbus OH 43210 USA. He is now with the GM R\&D Center, Warren, MI 48090-9055 USA (e-mail: quet.1@osu.edu).

H. Özbay is with the Department Electrical and Electronics Engineering, Bilkent University, Bilkent, Ankara TR-06800, Turkey, on leave from the Department of Electrical and Computer Engineering, The Ohio State University, Columbus OH 43210 USA (e-mail: ozbay.1@ osu.edu).

Digital Object Identifier 10.1109/TAC.2004.829643

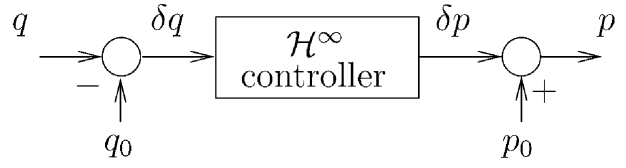

Fig. 1. AQM implementation.

mechanisms, thus providing a model and analysis during the congestion avoidance mode only. In TCP, the congestion window size $(W(t))$ is increased by one every round trip time if no congestion is detected, and is halved upon a congestion detection. This additive-increase multiplicative-decrease behavior of TCP has been modeled in [1] by the following difference equation (case of one TCP flow interacting with a single router):

$$
d W(t)=\frac{d t}{R(t)}-\frac{W(t)}{2} d N(t)
$$

with $R(t)=q(t) / C+T_{p}$ where $T_{p}$ is the propagation delay, $q(t)$ is the queue length at the router, $C$ is the router's transmission capacity, thus, $q(t) / C$ is the queuing delay and $R(t)$ is the round trip time delay, and $d N(t)$ is the number of marks the flow suffers. In a network topology of $N$ homogeneous TCP sources and one router, a model relating the average value of these variables and the router's queue dynamics becomes [2]

$$
\begin{aligned}
\dot{W}(t) & =\frac{1}{R(t)}-\frac{W(t)}{2} \frac{W(t-R(t))}{R(t-R(t))} p(t-R(t)) \\
\dot{q}(t) & =\left[\frac{N(t)}{R(t)} W(t)-C\right]^{+}
\end{aligned}
$$

where $p(t)$ is the probability of packet mark due to the AQM mechanism at the router. Here we use the notation $[x]^{+}=x$ if $x \geq 0$, and $[x]^{+}=0$ if $x<0$.

The linearization of (2) and (3) about the operating point is carried out in [2] and the perturbed variables about the operating point satisfy

$$
\begin{aligned}
\delta \dot{W}(t)= & -\frac{N}{R_{0}^{2} C}\left(\delta W(t)+\delta W\left(t-R_{0}\right)\right) \\
& -\frac{1}{R_{0}^{2} C}\left(\delta q(t)-\delta q\left(t-R_{0}\right)\right)-\frac{R_{0} C^{2}}{2 N^{2}} \delta p\left(t-R_{0}\right) \\
\dot{\delta} q(t)= & \frac{N}{R_{0}} \delta W(t)-\frac{1}{R_{0}} \delta q(t)
\end{aligned}
$$

where the operating point is defined by the solution $\left(R_{0}, W_{0}, p_{0}\right)$ of the following set of equations:

$$
\begin{aligned}
R_{0} & =\frac{q_{0}}{C}+T_{p} \\
W_{0} & =\frac{R_{0} C}{N} \\
p_{0} & =\frac{2}{W_{0}^{2}}
\end{aligned}
$$

for a desired equilibrium queue level $q_{0}$. Then, $W(t)=W_{0}+\delta W(t)$, and similarly for $R(t), p(t), q(t)$. Clearly, implementation of the controller depends on $q_{0}$, see Fig. 1. In the random early detection (RED) algorithm, $q_{0}$ is adjusted by setting appropriate parameters to satisfy

$$
p_{0}=L_{\mathrm{RED}}\left(q_{0}-\min _{\mathrm{th}}\right)
$$

where $L_{\mathrm{RED}}$ and $\min _{\text {th }}$ are the AQM-RED parameters $\left(L_{\mathrm{RED}}\right.$ is the ratio of a small change in packet mark probability to a small change in queue length, and $\min _{\text {th }}$ is the minimum queue length beyond which 
packet marking is applied linearly), see [1], [2]. Thus, (6)-(9) define the operating point that is adjusted by the RED parameters. Then, around the operating point, RED can be seen as a linear proportional controller with gain $L_{\mathrm{RED}}$.

Here, we consider the same linear plant derived in [2]. Note that for the linearization the time-varying nature of the roundtrip time delay in the terms " $t-R(t)$ " is ignored and these terms are approximated by " $t-R_{0}$." However, the queue length still depends on the round-trip time in the dynamical equation (3).

From (4) and (5) we derive the transfer function from $\delta p$ to $\delta q$

$$
\frac{\delta q(s)}{\delta p(s)}=-\frac{N W_{0}^{3}}{2} \frac{A(s) \mathrm{e}^{-R_{0} s}}{1+A(s) R_{0} s \mathrm{e}^{-R_{0} s}}
$$

where

$$
A(s)=\frac{1}{W_{0}\left(R_{0} s\right)^{2}+\left(W_{0}+1\right) R_{0} s+2} .
$$

Considering a negative feedback control system with the AQM being the controller, the system to be controlled is given by

$$
\begin{aligned}
P(s) & =\frac{N W_{0}^{3}}{2} \frac{A(s) \mathrm{e}^{-R_{0} s}}{1+A(s) R_{0} s \mathrm{e}^{-R_{0} s}} \\
& =\frac{N W_{0}^{3}}{2} \frac{\mathrm{e}^{-R_{0} s}}{\frac{R_{0} C}{N} R_{0}^{2} s^{2}+\left(\frac{R_{0} C}{N}+1\right) R_{0} s+2+R_{0} s \mathrm{e}^{-R_{0} s}} .
\end{aligned}
$$

Lemma 2.1: The plant $P$ defined in (11) is stable for all positive values of $R_{0}, C$, and $N$.

Proof: The poles of the transfer function $A$ are in the left-half part of the complex plane for all values of the parameters $W_{0}$ and $R_{0}$ positives, thus $A$ is always stable. We also have

$$
\begin{aligned}
& \left|A(s) R_{0} s \mathrm{e}^{-R_{0} s}\right|_{s=j \omega} \\
& \quad=\frac{R_{0} \omega}{\sqrt{\left(2-W_{0} R_{0}^{2} \omega^{2}\right)^{2}+\left(W_{0}+1\right)^{2}\left(R_{0} \omega\right)^{2}}} \\
& \quad<1
\end{aligned}
$$

for all positive values of the parameters $W_{0}$ and $R_{0}$. So according to the Nyquist stability test the transfer function

$$
\frac{1}{1+A(s) R_{0} s \mathrm{e}^{-R_{0} s}}
$$

is stable for all positive $W_{0}$ and $R_{0}$. We can thus conclude that the plant $P$ defined in (11) is stable for all positive values of $R_{0}, C$, and $N$.

In the following, we design an AQM scheme based on $\mathcal{H}^{\infty}$ control techniques that improves the system's transient while stabilizing the plant, and ensures robustness with respect to uncertainties in the values of the system's parameters.

\section{Modeling of the Parametric UnCertainties of the Plant}

Note that $P$ in $(11)$ can be written as

$$
P(s)=P_{2}(s) \frac{P_{1}(s)}{1+P_{1}(s)}
$$

with

$$
P_{1}(s)=A(s) R_{0} s \mathrm{e}^{-R_{0} s}
$$

and

$$
P_{2}(s)=\frac{N W_{0}^{3}}{2 R_{0} s}
$$

We assume that the parameters have known nominal values $N_{n}, R_{0 n}, C_{n}, W_{0 n}=\left(R_{0 n} C_{n} / N_{n}\right)$ and that we have the following bounds for their uncertainty:

$$
\begin{aligned}
\left|N-N_{n}\right| & \leq \Delta N^{+} \\
\left|R_{0}-R_{0 n}\right| & \leq \Delta R_{0}^{+} \\
\left|C-C_{n}\right| & \leq \Delta C^{+} .
\end{aligned}
$$

Assuming that $\left(R_{0 n}-\Delta R_{0}^{+}\right),\left(C_{n}-\Delta C^{+}\right),\left(N_{n}-\Delta N^{+}\right)$are positives, we also have

$$
\left|W_{0}-W_{0 n}\right| \leq \Delta W_{0}^{+}
$$

where

$$
\Delta W_{0}^{+}=\max \left\{\begin{array}{l}
\frac{\left(R_{0 n}+\Delta R_{0}^{+}\right)\left(C_{n}+\Delta C^{+}\right)}{\left(N_{n}-\Delta N+\right)}-W_{0 n} \\
W_{0 n}-\frac{\left(R_{0 n}-\Delta R_{0}^{+}\right)\left(C_{n}-\Delta C^{+}\right)}{\left(N_{n}+\Delta N^{+}\right)}
\end{array}\right\} .
$$

The plant $P$ can be written as

$$
P(s)=P_{n}(s)(1+\Delta P(s))
$$

where $P_{n}(s)$ is the nominal plant $(P(s)$ defined from the nominal values of the parameters $\left.N, R_{0}, C\right)$ and $\Delta P(s)$ is the multiplicative plant uncertainty. We would like to design a controller for the nominal plant $P_{n}(s)$ that would also stabilize the actual plant $P(s)$ that would be obtained by the same linearization process around the actual equilibrium point. In the following our goal is to find a bound $W_{2}(s)$ for the multiplicative plant uncertainty $\Delta P(s)$. For clarity of presentation we omit in the following the argument of the transfer functions (i.e., we just write $P$ instead of $P(s)$ ). Note that

$$
\begin{aligned}
& \frac{P_{1 n}+\Delta P_{1}}{1+P_{1 n}+\Delta P_{1}}\left(P_{2 n}+\Delta P_{2}\right) \\
& =\frac{P_{1 n} P_{2 n}}{1+P_{1 n}}\left(\frac{1}{1+\frac{\Delta P_{1}}{1+P_{1 n}}}+\frac{\frac{\Delta P_{2}}{P_{2 n}}}{1+\frac{\Delta P_{1}}{1+P_{1 n}}}+\frac{\frac{\Delta P_{1}\left(P_{2 n}+\Delta P_{2}\right)}{P_{1 n} P_{2 n}}}{1+\frac{\Delta P_{1}}{1+P_{1 n}}}\right) \\
& =\frac{P_{1 n} P_{2 n}}{1+P_{1 n}}\left(1+\frac{-\frac{\Delta P_{1}}{1+P_{1 n}}+\frac{\Delta P_{2}}{P_{2 n}}+\frac{\Delta P_{1}\left(P_{2 n}+\Delta P_{2}\right)}{P_{1 n} P_{2 n}}}{1+\frac{\Delta P_{1}}{1+P_{1 n}}}\right)
\end{aligned}
$$

so

$$
\begin{aligned}
\Delta P= & \frac{-\frac{\Delta P_{1}}{1+P_{1 n}}+\frac{\Delta P_{2}}{P_{2 n}}+\frac{\Delta P_{1}\left(P_{2 n}+\Delta P_{2}\right)}{P_{1 n} P_{2 n}}}{1+\frac{\Delta P_{1}}{1+P_{1 n}}} \\
= & \frac{1}{1+P_{1 n}+\Delta P_{1}} \cdot\left(\frac{\Delta P_{1}}{P_{1 n}}+\frac{\Delta P_{2}}{P_{2 n}}\left(1+P_{1 n}\right)\right. \\
& \left.+\frac{\Delta P_{1}}{P_{1 n}} \frac{\Delta P_{2}}{P_{2 n}}\left(1+P_{1 n}\right)\right) .
\end{aligned}
$$

It can be shown that a bound $W_{2}$ of the multiplicative plant uncertainty, i.e., $W_{2}$ that satisfies

$$
|\Delta P(s)|_{s=j \omega} \leq\left|W_{2}(s)\right|_{s=j \omega} \quad \forall \omega \in \mathbb{R}^{+}
$$

is (see details of the derivation in the Appendix)

$$
W_{2}(s)=a+b s+c s^{2}
$$

with $a, b$, and $c$ defined in (38)-(40).

\section{IV. $\mathcal{H}^{\infty}$ OPTIMIZATION PROBLEM}

We design the controller to minimize the following $\mathcal{H}^{\infty}$ cost function:

$$
\inf \left\|\left[\begin{array}{c}
W_{1}\left(1+P_{n}(s) C(s)\right)^{-1} \\
W_{2} P_{n}(s) C(s)\left(1+P_{n}(s) C(s)\right)^{-1}
\end{array}\right]\right\|_{\infty}=: \gamma_{\mathrm{opt}}
$$


where the infimum is taken over all $C$ stabilizing $P_{n}$, and $W_{1}(s)=1 / s$ for good tracking of step-like reference inputs (desired queue size). Applying the formulas given in [12] and [13], the optimal solution to (20) is found as (a similar derivation can be found in [14])

$$
\begin{aligned}
C_{\mathrm{opt}}(s)= & \frac{2 \gamma}{c N_{n} W_{0 n}^{3}} \\
& \times \frac{W_{0 n} R_{0 n}^{2} s^{2}+\left(W_{0 n}+1\right) R_{0 n} s+2+R_{0 n} s e^{-R_{0 n} s}}{s^{2}} \\
& \cdot \frac{1}{1+\frac{a_{2} c_{2} \gamma^{2}}{s}+F(s)}
\end{aligned}
$$

where $F$ is a finite impulse response filter defined as

$$
f(t)= \begin{cases}\left(b_{2}+a_{2}-a_{2} c_{2} \gamma^{2}\right) \cos \left(\frac{t}{\gamma}\right) & \\ \quad+\gamma\left(c_{2}+a_{2} b_{2}-\frac{1}{\gamma^{2}}\right) \sin \left(\frac{t}{\gamma}\right), & \text { for } t<R_{0 n} \\ 0, & \text { otherwise }\end{cases}
$$

with

$$
\begin{aligned}
& a_{2}=\frac{1}{c \gamma} \sqrt{\frac{\gamma^{2}-a^{2}}{x}} \\
& b_{2}=\sqrt{\frac{\left(b^{2}-2 a c\right) \gamma^{2}-c^{2}}{c^{2} \gamma^{2}}+2 \sqrt{x}-\frac{\gamma^{2}-a^{2}}{c^{2} \gamma^{2} x}} \\
& c_{2}=\sqrt{x}
\end{aligned}
$$

where $x$ is the unique positive root of

$$
\begin{aligned}
x^{3}+\frac{b^{2}-2 a c-a^{2} \gamma^{2}}{c^{2} \gamma^{2}} x^{2}-\frac{\left(\gamma^{2}-a^{2}\right)\left[\left(2 a c-b^{2}\right) \gamma^{2}+c^{2}\right]}{c^{4} \gamma^{4}} & x \\
-\frac{\left(\gamma^{2}-a^{2}\right)^{2}}{c^{4} \gamma^{4}} & =0
\end{aligned}
$$

and $\gamma$ is determined as the largest root of

$$
1-\left.\frac{\gamma}{c} \mathrm{e}^{-R_{0 n} s} \frac{s}{\left(s+a_{2}\right)\left(s^{2}+b_{2} s+c_{2}\right)}\right|_{s=\frac{j}{\gamma}}=0 .
$$

\section{IMPLEMENTATION ISSUES}

The packets are marked with probability $p(t)$ computed by the $\mathcal{H}^{\infty}$ controller (21) according to the block diagram shown in Fig. 1 where $q_{0}$ is the desired steady-state queue length and

$$
p_{0}=\frac{2 N_{n}^{2}}{\left(\frac{q_{0}}{C_{n}}+T_{p n}\right)^{2} C_{n}^{2}}
$$

is calculated from (6)-(8).

\section{SimUlationS}

The simulations are carried out using simulink, the nonlinear model defined by (2) and (3) representing the dynamics of $N$ TCP flows loading a router. The router implements the AQM scheme defined by (21). The following scenario is considered.

- Nominal values known to the controller: $N_{n}=50$ TCP sessions, $C_{n}=300$ packets/s, $T_{p}=0.2 \mathrm{~s}$, so $R_{0 n}=0.533 \mathrm{~s}$ and $W_{0 n}=$ 3.2 packets (we assume here a fluid model and, thus, we do not consider packetization issues).

- Real values of the plant: $N=40$ TCP sessions, $C=250$ packets $/ \mathrm{s}, T_{p}=0.3 \mathrm{~s}$, so $R_{0}=0.7 \mathrm{~s}$ and $W_{0}=4.375$ packets.

- The following controller design parameters are considered: $\Delta N^{+}=10, \Delta R_{0}^{+}=0.1, \Delta C^{+}=50$, which implies $\Delta W_{0}^{+}=2.3417$.

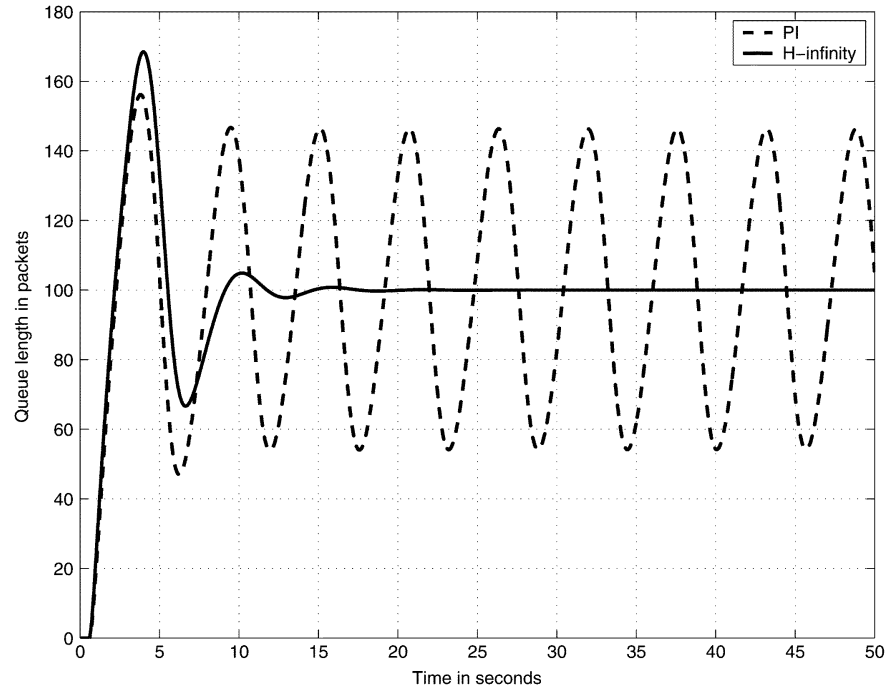

Fig. 2. Comparison between $\mathcal{H}^{\infty}$ and PI controllers.

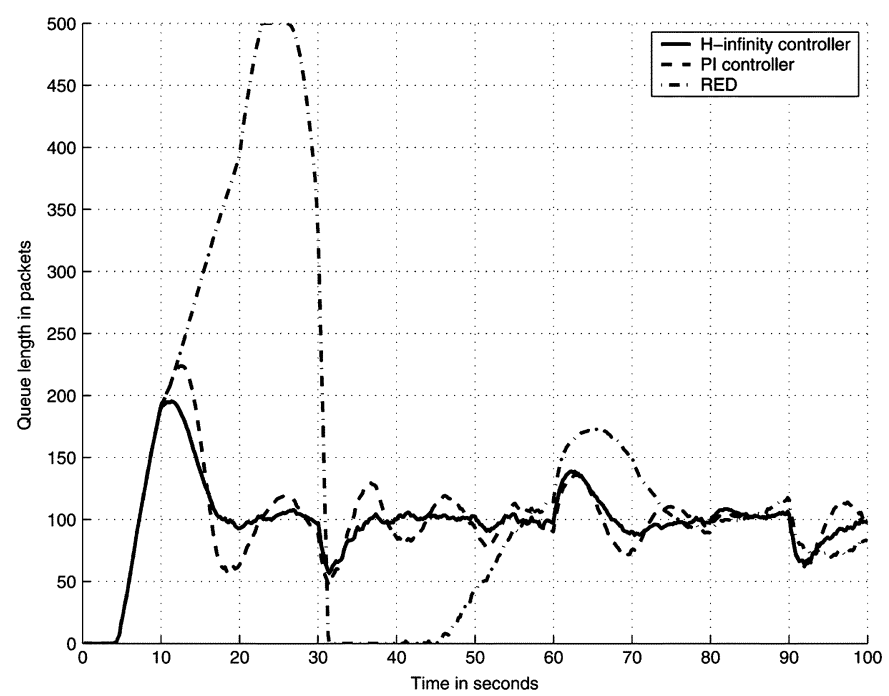

Fig. 3. Comparison between $\mathcal{H}^{\infty}$, PI controllers and RED.

For comparison we also simulate the PI AQM scheme proposed in [2]. The parameters of the PI controller are computed as suggested by the authors of [2] using the aforementioned known nominal values. We can see in Fig. 2 that the $\mathcal{H}^{\infty}$ controller performs significantly better than the PI controller for this set of parameters.

In Figs. 3 and 4, we analyze the robustness of the two schemes with respect to variations in the network parameters. The outgoing link capacity $C$ is a normally distributed random signal with mean 250 packets/s and variance 50 added to a pulse of period $60 \mathrm{~s}$, amplitude 60 packets/s. The number of TCP flows $N$ is a normally distributed random signal with mean 45 and variance 30 added to a pulse of period $20 \mathrm{~s}$ and amplitude 10 . The propagation delay $T_{p}$ is a normally distributed random signal with mean $0.8 \mathrm{~s}$ and variance $0.05 \mathrm{~s}$ added to a pulse of period $20 \mathrm{~s}$ and amplitude $0.2 \mathrm{~s}$. The controllers have the following value known to them: $C=300$ packets/s, $N=50, T_{p}=$ $0.7 \mathrm{~s}$ and the desired queue length is $q_{0}=100$ packets. In addition the $\mathcal{H}^{\infty}$ controller uses the following design parameters: $\Delta N^{+}=$ $10, \Delta R_{0}^{+}=0.1$, and $\Delta C^{+}=50$. RED [15] has the following parameters: $p_{\max }=0.1, \min _{\text {th }}=80, \max _{\text {th }}=150$, (these determine the AQM-RED controller gain as $\left.L_{\text {RED }}=\left(p_{\max }\right) /\left(\max _{\text {th }}-\min _{\text {th }}\right)\right)$ and queue averaging weight $=0.0001$. It can be seen that both the 

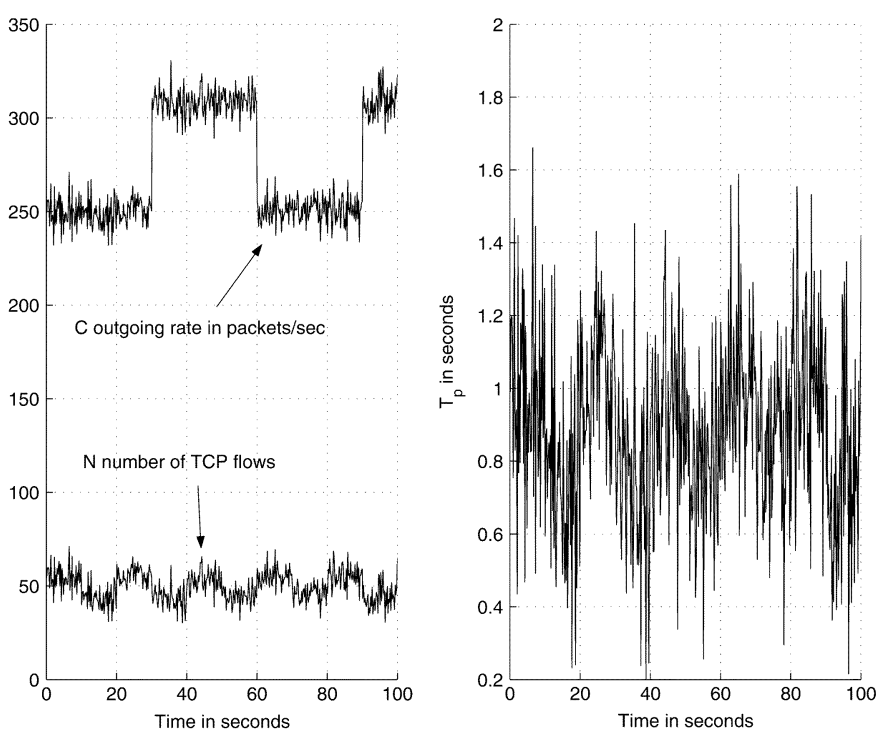

Fig. 4. Values of $\boldsymbol{C}, \boldsymbol{N}$, and $\boldsymbol{T}_{p}$ corresponding to Fig. 3.

PI and the $\mathcal{H}^{\infty}$ controllers perform better than RED as the initial overshoot is reduced, and the system oscillates less under parameter variations. The wide oscillations of RED can cause the queue to become empty, thus decreasing the utilization of the outgoing link.

\section{CONCLUDING REMARKS}

We developed in this note a robust AQM control scheme supporting ECN and TCP flows. The simulation experiments based on a nonlinear fluid flow model, that includes delays, show that the proposed AQM scheme performs better than RED and the proportional-integral scheme by obtaining faster transients and less oscillatory responses, which translates into higher link utilization, low packet loss rate and small queue fluctuations. A challenging extension of this work would be to consider networks having multiple links and sources, but such an extension is not trivial due to the interaction between the control schemes of each router.

\section{APPENDIX}

From (17), we see that we can bound the multiplicative plant uncertainty in the following way:

$$
\begin{aligned}
|\Delta P(s)|_{s=j \omega} & \frac{1}{1-\text { upper bound on }\left|P_{1 n}+\Delta P_{1}\right|_{s=j \omega}} \\
& \cdot\left(\left|\frac{\Delta P_{1}}{P_{1 n}}\right|_{s=j \omega}+\left|\frac{\Delta P_{2}}{P_{2 n}}\left(1+P_{1 n}\right)\right|_{s=j \omega}\right. \\
& \left.+\left|\frac{\Delta P_{1}}{P_{1 n}}\right|_{s=j \omega} \cdot\left|\frac{\Delta P_{2}}{P_{2 n}}\left(1+P_{1 n}\right)\right|_{s=j \omega}\right) .
\end{aligned}
$$

Note that

$$
\frac{1}{W_{0 n} R_{0 n}^{2} s^{2}+\left(W_{0 n}+1\right) R_{0 n} s+2}=\frac{\frac{1}{W_{0 n} R_{0 n}^{2}}}{s^{2}+2 \xi \omega_{0}+\omega_{0}^{2}}
$$

with

$$
\omega_{0}=\frac{1}{R_{0 n}} \sqrt{\frac{2}{W_{0 n}}} \quad \xi=\frac{W_{0 n}+1}{2 \sqrt{2 W_{0 n}}} .
$$

We have

$$
W_{0 n}+1 \geq 2 \sqrt{W_{0 n}} \quad \forall W_{0 n}
$$

thus

$$
\xi \geq \frac{1}{\sqrt{2}} \quad \forall W_{0 n}
$$

and, consequently, the transfer function (30) do not exhibit any resonance phenomenon and

$$
\begin{aligned}
& \left|\frac{1}{W_{0 n} R_{0 n}^{2} s^{2}+\left(W_{0 n}+1\right) R_{0 n} s+2}\right|_{s=j \omega} \\
& \quad \leq\left|\frac{1}{W_{0 n} R_{0 n}^{2} s^{2}+\left(W_{0 n}+1\right) R_{0 n} s+2}\right|_{s=0} \\
& \quad \leq \frac{1}{2} .
\end{aligned}
$$

$$
\begin{aligned}
\Delta P_{2}(s) & =P_{2 n}(s)+\Delta P_{2}(s)-P_{2 n}(s) \\
& =\frac{N_{n} W_{0 n}^{3}+N_{n}\left(3 W_{0 n}^{2} \Delta W_{0}+3 W_{0 n} \Delta W_{0}^{2}+\Delta W_{0}^{3}\right)+\Delta N\left(W_{0 n}+\Delta W_{0}\right)^{3}}{2 R_{0 n} s\left(1+\frac{\Delta R_{0}}{R_{0 n}}\right)}-\frac{N_{n} W_{0 n}^{3}}{2 R_{0 n} s} \\
& =\frac{N_{n}\left(3 W_{0 n}^{2} \Delta W_{0}+3 W_{0 n} \Delta W_{0}^{2}+\Delta W_{0}^{3}\right)+\Delta N\left(W_{0 n}+\Delta W_{0}\right)^{3}-N_{n} W_{0 n}^{3} \frac{\Delta R_{0}}{R_{0 n}}}{2 R_{0 n} s\left(1+\frac{\Delta R_{0}}{R_{0 n}}\right)}
\end{aligned}
$$

$$
\begin{aligned}
\left|\frac{\Delta P_{1}}{P_{1 n}}\right|_{s=j \omega} \\
=\left|1-\frac{P_{1 n}+\Delta P_{1}}{P_{1 n}}\right|_{s=j \omega} \\
=\mid 1-\frac{R_{0 n}+\Delta R_{0}}{R_{0 n}} \cdot \mathrm{e}^{-\Delta R_{0} s} \\
\left.\quad \cdot \frac{W_{0 n} R_{0 n}^{2} s^{2}+\left(W_{0 n}+1\right) R_{0 n} s+2}{\left(W_{0 n}+\Delta W_{0}\right)\left(R_{0 n}+\Delta R_{0}\right)^{2} s^{2}+\left(W_{0 n}+\Delta W_{0}+1\right)\left(R_{0 n}+\Delta R_{0}\right) s+2}\right|_{s=j \omega} \\
\leq 1+\left(1+\frac{\Delta R_{0}^{+}}{R_{0 n}}\right) \frac{1}{2}\left|W_{0 n} R_{0 n}^{2} s^{2}+\left(W_{0 n}+1\right) R_{0 n} s+2\right|_{s=j \omega} \cdot
\end{aligned}
$$




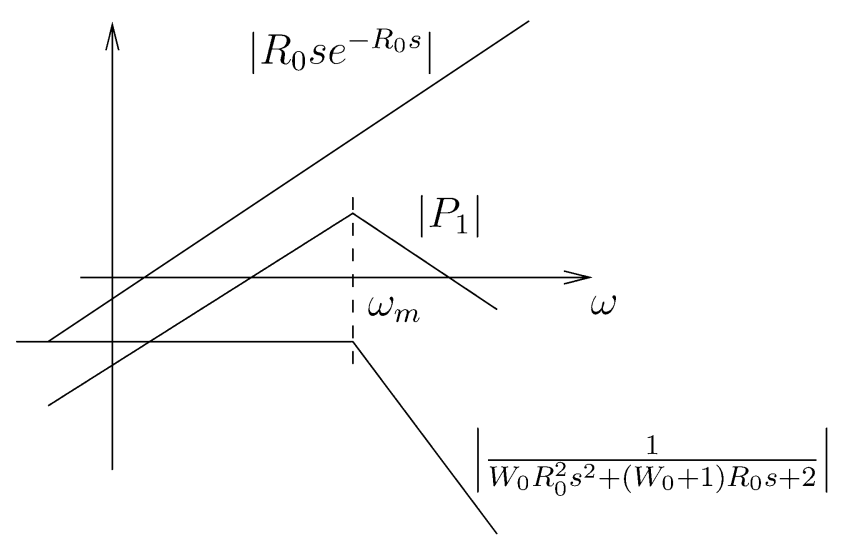

Fig. 5. Case $\Delta<0$.

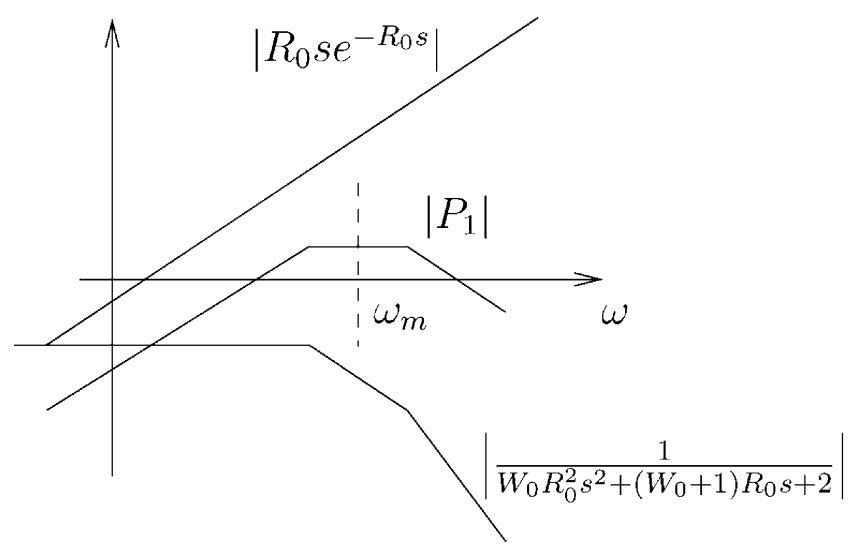

Fig. 6. Case $\Delta>0$.

Similarly, we have

$$
\begin{aligned}
\mid\left(W_{0 n}+\Delta W_{0}\right) & \left(R_{0 n}+\Delta R_{0}\right)^{2} s^{2} \\
& +\left(W_{0 n}+\Delta W_{0}+1\right)\left(R_{0 n}+\Delta R_{0}\right) s+\left.2\right|_{s=j \omega} \geq 2 .
\end{aligned}
$$

The discriminant of the second order polynomial in $s$ formed by the denominator of the transfer function $P_{1}(s)$ is

$$
\begin{aligned}
\Delta & =\left(W_{0}+1\right)^{2} R_{0}^{2}-8 W_{0} R_{0}^{2} \\
& =R_{0}^{2}\left(W_{0}^{2}-6 W_{0}+1\right) .
\end{aligned}
$$

A sketch of the bode plot of $P_{1}$ is shown in Figs. 5, and 6. For the case of $\Delta<0$ corresponding to Fig. 5 an upper bound for the magnitude of $P_{1}(s)$ evaluated on the imaginary axis is $\left|P_{1}\left(\omega_{m}\right)\right|$ where $\omega_{m}=$ $\left(1 / R_{0}\right) \sqrt{\left(2 / W_{0}\right)}$. For the case of $\Delta>0$ corresponding to Fig. 6 an upper bound for the magnitude of $P_{1}(s)$ evaluated on the imaginary axis is $\left|P_{1}\left(\omega_{m}\right)\right|$ where $\omega_{m}$ is the geometric mean between the roots of the second-order polynomial in $s$ formed by the denominator of the transfer function $P_{1}(s)$, which is also $\omega_{m}=\left(1 / R_{0}\right) \sqrt{\left(2 / W_{0}\right)}$. Thus, we have

$$
\begin{aligned}
\left|P_{1 n}+\Delta P_{1}\right|_{s=j \omega} & <\max _{\Delta W_{0}, \Delta R_{0}}\left(\frac{R_{0 n}+\Delta R_{0}}{2} \cdot \omega_{m}\right) \\
& <\max _{\Delta W_{0}}\left(\frac{1}{2} \sqrt{\frac{2}{W_{0 n}+\Delta W_{0}}}\right) .
\end{aligned}
$$

Assuming $W_{0 n}+\Delta W_{0}>1$, we have

$$
\left|P_{1 n}+\Delta P_{1}\right|_{s=j \omega}<\frac{\sqrt{2}}{2} .
$$

We also have the first equation shown at the bottom of the previous page, as well as (32), also shown at the bottom of the previous page, and a bound of the magnitude of $\Delta P_{2}(s)$ on the imaginary axis is

$$
\left|\Delta P_{2}(s)\right|_{s=j \omega}<\left|\frac{Z_{2}}{s}\right|_{s=j \omega}
$$

with

$$
\begin{gathered}
Z_{2}=\frac{N_{n}\left(3 W_{0 n}^{2} \Delta W_{0}^{+}+3 W_{0 n}\left(\Delta W_{0}^{+}\right)^{2}+\left(\Delta W_{0}^{+}\right)^{3}\right)}{2 R_{0 n}\left(1-\frac{\Delta R_{0}^{+}}{R_{0 n}}\right)} \\
+\frac{\Delta N^{+}\left(W_{0 n}+\Delta W_{0}^{+}\right)^{3}+N_{n} W_{0 n}^{3} \frac{\Delta R_{0}^{+}}{R_{0 n}}}{2 R_{0 n}\left(1-\frac{\Delta R_{0}^{+}}{R_{0 n}}\right)}
\end{gathered}
$$

so we have

$$
\left|\frac{\Delta P_{2}}{P_{2 n}}\right|_{s=j \omega}<\frac{2 R_{0 n} Z_{2}}{N_{n} W_{0 n}^{3}} .
$$

We also have

$$
\begin{aligned}
\mid 1 & +\left.P_{1 n}\right|_{s=j \omega} \leq 1 \\
& +\left|\frac{R_{0 n} s}{W_{0 n} R_{0 n}^{2} s^{2}+\left(W_{0 n}+1\right) R_{0 n} s+2}\right|_{s=j \frac{1}{R_{0 n}} \sqrt{\frac{2}{W_{0 n}}}} \\
\leq & 1+\frac{1}{W_{0 n}+1} \\
\leq & \frac{3}{2}
\end{aligned}
$$

if we assume $W_{0 n}>1$.

With (31)-(34), (29) can be written as

$$
|\Delta P(s)|_{s=j \omega}<\tilde{W}_{2}(\omega)
$$

with

$$
\begin{aligned}
\tilde{W}_{2}(\omega)= & \frac{1}{1-\frac{\sqrt{2}}{2}}\left\{\left[1+\frac{1}{2}\left(1+\frac{\Delta R_{0}^{+}}{R_{0 n}}\right)\right.\right. \\
& \left.\cdot \sqrt{\left(2-W_{0 n} R_{0 n}^{2} \omega^{2}\right)^{2}+\left(W_{0 n}+1\right)^{2} R_{0 n}^{2} \omega^{2}}\right] \\
& \left.\cdot\left(1+\frac{3 R_{0 n} Z_{2}}{N_{n} W_{0 n}^{3}}\right)+\frac{3 R_{0 n} Z_{2}}{N_{n} W_{0 n}^{3}}\right\} \\
= & a_{3}+\sqrt{b_{3}+c_{3} \omega^{2}+d_{3} \omega^{4}}
\end{aligned}
$$

and

$$
\begin{aligned}
a_{3} & =\frac{1}{1-\frac{\sqrt{2}}{2}}\left\{1+\frac{6 R_{0 n} Z_{2}}{N_{n} W_{0 n}^{3}}\right\} \\
e_{3} & =\frac{1}{4}\left(\frac{1}{1-\frac{\sqrt{2}}{2}}\right)^{2}\left(1+\frac{\Delta R_{0}^{+}}{R_{0 n}}\right)^{2}\left(1+\frac{3 R_{0 n} Z_{2}}{N_{n} W_{0 n}^{3}}\right)^{2} \\
b_{3} & =4 e_{3} \\
c_{3} & =\left(W_{0 n}^{2}+R_{0 n}^{2}-2 W_{0 n} R_{0 n}^{2}\right) e_{3} \\
d_{3} & =W_{0 n}^{2} R_{0 n}^{4} e_{3} .
\end{aligned}
$$

Note that we have

$$
\tilde{W}_{2}(\omega)<\left|W_{2}(s)\right|_{s=j \omega}
$$

with

$$
W_{2}(s)=a+b s+c s^{2}
$$


where

$$
\begin{aligned}
& a=a_{3}+\sqrt{b_{3}} \\
& c=\sqrt{d_{3}} \\
& b=\max \left\{\sqrt{2 a_{3} \sqrt{d_{3}}+2 a c+c_{3}}, \sqrt{\frac{a_{3} c_{3}}{\sqrt{b_{3}}}+2 a c+c_{3}}\right\} .
\end{aligned}
$$

\section{REFERENCES}

[1] V. Misra, W. B. Gong, and D. Towsley, "Fluid-based analysis of a network of AQM routers supporting TCP flows with an application to RED," in Proc. ACM/SIGCOMM, 2000, pp. 151-160.

[2] C. V. Hollot, V. Misra, D. Towsley, and W. B. Gong, "Analysis and design of controllers for AQM routers supporting TCP flows," IEEE Trans. Automat. Contr., vol. 47, pp. 945-959, June 2002.

[3] F. Kelly, "Mathematical modeling of the internet," in Mathematics Unlimited-2001 and Beyond, B. Engquist and W. Schmid, Eds. Berlin, Germany: Springer-Verlag, 2001

[4] T. Ott, T. Lakshman, and L. Wong, "SRED: Stabilized RED," in Proc. IEEE INFOCOM, 1999.

[5] S. Low, F. Paganini, J. Wang, S. Adlakha, and J. Doyle, "Dynamics of TCP/RED and a scalable control," in Proc. IEEE INFOCOM'02, New York, June 2002.
[6] S. Kunniyur and R. Srikant, "Analysis and design of an adaptive virtual queue algorithm for active queue management," in Proc. SIGCOMM 2001, Aug. 2001.

[7] R. Johari and D. K. H. Tan, "End-to-end congestion control for the internet: Delays and stability," IEEE/ACM Trans. Networking, vol. 9, pp. $818-832$, Dec. 2001, to be published.

[8] L. Massoulie, "Stability of distributed congestion control with heterogeneous feedback delays," Microsoft Research Tech. Rep., 2000.

[9] G. Vinnicombe, "On the stability of end-to-end congestion control for the internet," Univ. Cambridge Tech. Rep., 2001.

[10] S. H. Low, F. Paganini, and J. C. Doyle, "Internet congestion control," IEEE Contr. Syst. Mag., vol. 22, pp. 28-43, Jan. 2002.

[11] K. Ramakrishnan and S. Floyd, "A Proposal to add explicit congestion notification (ECN) to IP," RFC 2481, Jan. 1999.

[12] O. Toker and H. Özbay, " $\mathcal{H}^{\infty}$ optimal and suboptimal controllers for infinite dimensional SISO plants," IEEE Trans. Automat. Contr., vol. 40, pp. 751-755, June 1995.

[13] C. Foias, H. Özbay, and A. Tannenbaum, Robust Control of Infinite Dimensional Systems: Frequency Domain Methods. New York: Springer-Verlag, 1996.

[14] P.-F. Quet, B. Ataşlar, A. İftar, H. Özbay, T. Kang, and S. Kalyanaraman, "Rate-based flow controllers for communication networks in the presence of uncertain time-varying multiple time-delays," Automatica, vol. 38, pp. 917-928, June 2002.

[15] S. Floyd and V. Jacobson, "Random early detection gateways for congestion avoidance," IEEE/ACM Trans. Networking, vol. 1, pp. 397-413, Aug. 1993. 